\title{
EXPLOSIONS OF HELIUM STARS AND TYPE IB/IC/IIB SUPERNOVAE
}

\author{
K. NOMOTO \\ Department of Astronomy, Faculty of Science, University of Tokyo, Tokyo 113
}

\begin{abstract}
Theoretical models of supernova explosions of helium stars with various masses are reviewed to examine possible connections between Wolf-Rayet stars and Type $\mathrm{Ib} / \mathrm{Ic} / \mathrm{IIb}$ supernovae. Nucleosynthesis, Rayleigh-Taylor instabilities, and light curves are compared with observations. Maximum brightness and the fast decline of the light curves of typical SNe Ib/Ic can be well accounted for by the helium star models if the helium star mass is as low as 3-5 $M_{\odot}$. These low mass helium stars can form from stars of 12-18 $M_{\odot}$ after Roche-lobe overflow in close binary systems. Probably progenitors of typical SNe Ib/Ic are not classified as Wolf-Rayet stars.
\end{abstract}

\section{TYPE IB/IC SUPERNOVAE}

Supernova explosions of Wolf-Rayet stars may in principle be observed as Type I supernovae (SNe I) since their maximum light spectra must lack of hydrogen lines. In particular, Wolf-Rayet stars have been suggested to be promising candidates for the progenitors of Type Ib/Ic supernovae (SNe Ib/Ic), since most of SNe Ib are associated with star-forming regions (see, e.g., Harkness and Wheeler 1990 for a review and references).

Unlike the classical Type I supernovae (now designated as SNe Ia), SNe Ib exhibit strong absorption lines of $\mathrm{He} \mathrm{I}$ at early times, and prominent emission lines of [O I], [Ca II], and $\mathrm{Ca}$ II at late times. SNe Ic spectroscopically resemble $\mathrm{SNe} \mathrm{Ib}$, except that the He I lines are absent at early times (Harkness et al. 1987). The light curves of $\mathrm{SNe} \mathrm{Ib} / \mathrm{Ic}$, generally similar to SNe Ia, are very likely to be powered by the decays of ${ }^{56} \mathrm{Ni}$ and ${ }^{56} \mathrm{Co}$. Well-observed $\mathrm{SNe} \mathrm{Ib}$ are about $1.5 \mathrm{mag}$ fainter than $\mathrm{SNe}$ Ia at maximum (Uomoto and Kirshner 1985; Branch 1986; Panagia 1987).

These spectroscopic and photometric features have let to the Wolf-Rayet star models with a wide range of masses (Wheeler and Levreault 1985; Begelman and Sarazin 1986; Gaskel et al. 1986; Uomoto 1986; Schlegel and Kirshner 1989; Schaeffer, Casse, and Cahen 1987; Ensman and Woosley 1988; Nomoto et al. 1988a,b). 
However, the previous Wolf-Rayet star models have some difficulties 1) in reproducing the light curves of typical SNe Ib which decline as fast as SNe Ia (Panagia 1987; Leibundgut 1988), and 2) in producing enough ${ }^{56} \mathrm{Ni}$ to attain the maximum luminosities of $\mathrm{SNe} \mathrm{Ib}$ in relatively low mass helium star models (Ensman and Woosley 1988). In particular, the light curve of SN Ic 1987M has two important features that challenge the Wolf-Rayet models: (1) maximum brightness is only 0.6 mag dimmer than in SNe Ia, and significantly brighter than in typical SNe Ib; and (2) the decline of the light curve is faster than those of SNe Ia and SNe Ib (Filippenko et al. 1990; also SN Ic 1983I reported by Tsvetkov 1985).

Recently Shigeyama et al. (1990) have shown that the fast declines of the $\mathrm{SNe} \mathrm{Ib} / \mathrm{Ic}$ light curves are well reproduced if mixing of ${ }^{56} \mathrm{Ni}$ occurs in $3-5 M_{\odot}$ helium stars. Here we review nucleosynthesis, Rayleigh-Taylor instabilities, and light curves of exploding helium stars, and conclude that the helium stars of 3-5 $M_{\odot}$ (which form from stars with initial masses $M_{\mathrm{i}} \sim 12-18 M_{\odot}$ in binary systems) are the most likely progenitors of typical SNe Ib/Ic.

\section{NUCLEOSYNTHESIS IN EXPLODING HELIUM STARS AND THE MASS OF ${ }^{56} \mathrm{Ni}$}

In the helium star models for $\mathrm{SNe} \mathrm{Ib}$, two scenarios are possible for the presupernova evolution. 1) A fairly massive single star lost its hydrogen-rich envelope in a wind. 2) A star in a close binary system becomes a helium star by Roche-lobe overflow.

Shigeyama et al. (1990) adopted the second scenario and performed hydrodynamical calculations of the explosion of the helium stars of masses $M_{\alpha}=3.3$, 4 , and $6 M_{\odot}$, which are presumed to form from the main-sequence stars of masses $M_{\mathrm{i}} \sim$ 13,15 , and $20 M_{\odot}$, respectively. These stars eventually undergo iron core collapse as in Type II supernovae (SNe II). A shock wave is then formed at the mass cut that divides the neutron star and the ejecta, propagating outward to explosively synthesize ${ }^{56} \mathrm{Ni}$ and other heavy elements (e.g., Hashimoto et al. 1989; Thielemann et al. 1990).

Since the mechanism that transforms collapse into explosion is unclear, the mass cut and explosion energy are not known. The adopted presupernova models (Nomoto and Hashimoto 1988) have the following important difference from the previous models, i.e., the iron core masses are as small as $1.18 M_{\odot}$ and $1.28 M_{\odot}$ for $M_{\alpha}=$ $3.3 M_{\odot}$ and $4 M_{\odot}$, respectively, significantly smaller than $1.4 M_{\odot}$ in the $6 M_{\odot}$ star, due to the larger effect of Coulomb interactions during the progenitor's evolution. Because of steep density gradient at the outer edge of the iron core, it is reasonable to assume that the neutron star mass $M_{\mathrm{NS}}$ is equal to the iron core mass (Shigeyama et al. 1990). The final kinetic energy of explosion is assumed to be $E=1 \times 10^{51}$ erg. 


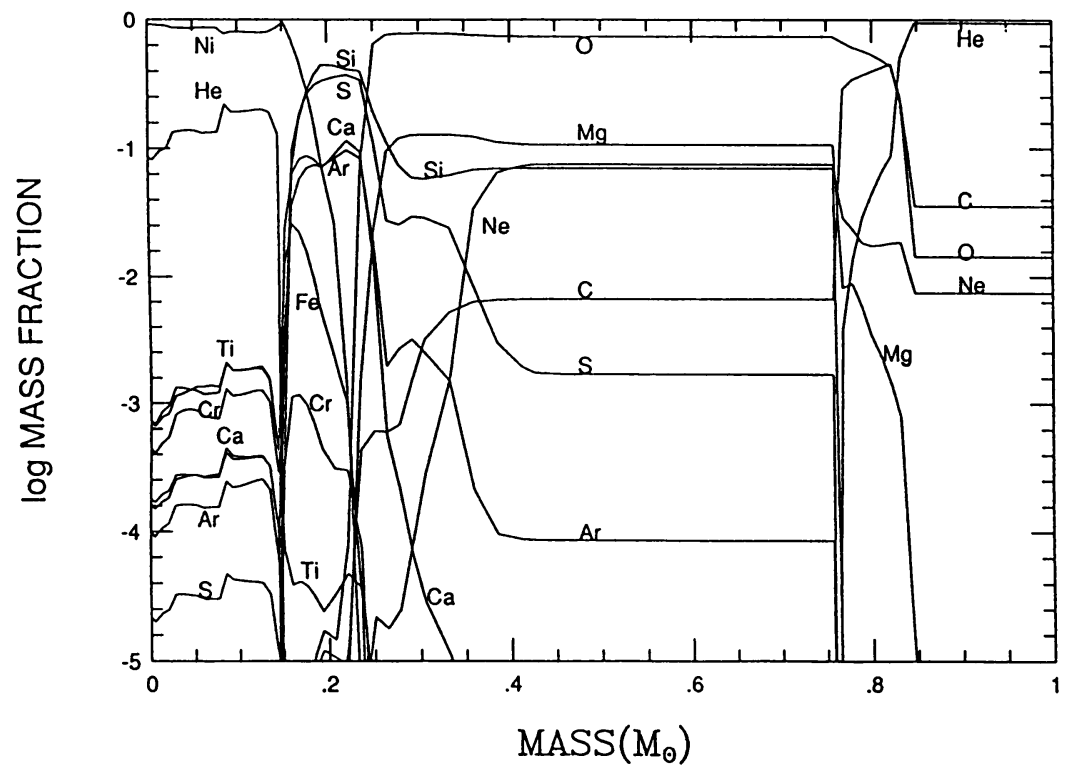

Fig. 1: Explosive nucleosynthesis in the $4 M_{\odot}$ helium star (Shigeyama et al. 1990). Composition of the innermost $1 M_{\odot}$ of the ejecta is shown. (The outermost $1.72 M_{\odot}$ helium layer and the $1.28 M_{\odot}$ neutron star are not included in the figure.) About $0.15 M_{\odot}{ }^{56} \mathrm{Ni}$ and $0.43 M_{\odot}$ oxygen are produced.

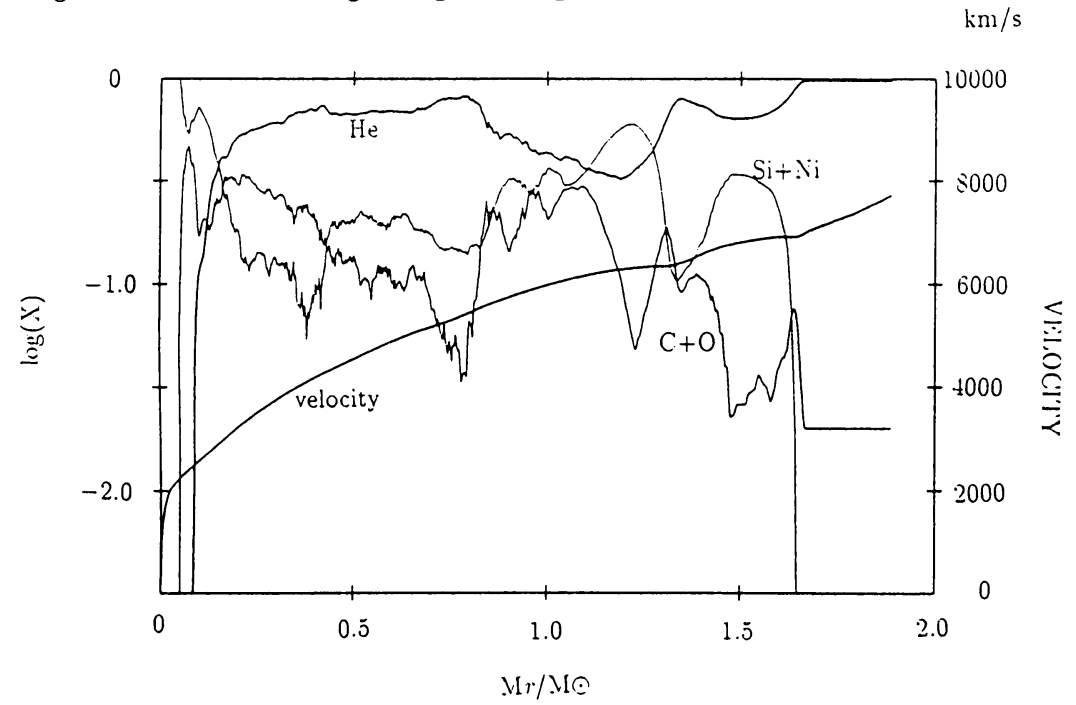

Fig. 3: Chemical composition for $M_{\alpha}=3 M_{\odot}$ at $t=180 \mathrm{~s}$ after explosion is plotted against the radial mass coordinate, $M_{r}$. Mass fractions of $\mathrm{He}, \mathrm{C}+\mathrm{O}$, and $\mathrm{Si}+\mathrm{Ni}$ are shown by the thick, intermediate, and the thin lines, respectively. The mean radial velocity is also shown (thick solid line). ${ }^{56} \mathrm{Ni}$ is mixed to the layer at $M_{r}=1.7 M_{\odot}$, i.e., $0.4 M_{\odot}$ beneath the surface (Hachisu et al. 1990b). 
Nucleosynthesis behind the shock front is determined by the temperature $T$, which is approximately given by $E=4 \pi r^{3} / 3 a T^{4}$ for the sphere of radius $r$ (e.g., Woosley 1988). For $T>5 \times 10^{9} \mathrm{~K}$, materials are processed into nuclear statistical equilibrium (NSE) composition, mostly ${ }^{56} \mathrm{Ni}$. This region corresponds to a sphere of radius $\sim 3700\left(E / 10^{51} \mathrm{erg}\right)^{1 / 3} \mathrm{~km}$ which contains a mass $M_{\mathrm{NSE}}(\sim 1.44-1.46$ $M_{\odot}$ for $M_{\alpha}=3.3$ and $\left.4 M_{\odot}\right)$. Therefore the mass of ${ }^{56} \mathrm{Ni}$ is approximately given by $M_{\mathrm{NSE}}-M_{\mathrm{NS}}$. Distribution of the nucleosynthesis products for $M_{\alpha}=4 M_{\odot}$ is shown in Fig. 1. The ${ }^{56} \mathrm{Ni}$ masses are 0.26 and $0.15 M_{\odot}$ for $M_{\alpha}=3.3$ and $4 M_{\odot}$, respectively, which are large enough to account for maximum brightness of SNe Ib. The oxygen masses are 0.21 and $0.43 M_{\odot}$ for $M_{\alpha}=3.3$ and $4 M_{\odot}$, respectively, and could be consistent with those inferred from the late time spectra of $\mathrm{SNe} \mathrm{Ib} / \mathrm{Ic}$ because the latter is sensitive to the temperature of the ejecta (e.g., Uomoto 1986).

The above results are in contrast to the previous models (Ensman and Woosley 1988 ) in which the mass of ${ }^{56} \mathrm{Ni}$ synthesized by helium stars decreases with decreasing progenitor mass. Given the constraint that SN $1987 \mathrm{~A}$ produced only $0.07 M_{\odot}$ of ${ }^{56} \mathrm{Ni}$ (e.g., Shigeyama et al. 1988; Woosley 1988), the helium star progenitor of SNe Ib, which produce $\sim 0.15 M_{\odot}{ }^{56} \mathrm{Ni}$ to attain the observed maximum luminosities, must have been more massive than $6 M_{\odot}$; this is incompatible with the requirement from the light curve shape $(\S 4)$.

\section{RAYLEIGH-TAYLOR INSTABILITIES AND MIXING}

As will be shown in $§ 5$, the helium star models are in good agreement with the observed $\mathrm{SNe} \mathrm{Ib}$ light curves only if the extensive mixing of ${ }^{56} \mathrm{Ni}$ takes place. Mixing and clumpiness in $\mathrm{SNe} \mathrm{Ib}$ is also inferred from the late time emission line features (Fransson and Chevalier 1989; Filippenko and Sargent 1989). Such a mixing of radioactive elements has been observed in SN 1987A (e.g., Kumagai et al. 1989 and references therein) and successfully accounted for by the Rayleigh-Taylor instability in the explosion (Arnett et al. 1989; Hachisu et al. 1990a; Den et al. 1990; Yamada et al. 1990; Fryxell et al. 1991).

The Rayleigh-Taylor instability in helium stars develop as follows. When the shock wave hits the helium envelope, the expansion of the inner core is largely decelerated, which forms a reverse shock. Then a pressure inversion appears (i.e., the pressure increases outward) in the layer between the forward shock and the reverse shock. The interface between the core and the helium envelope becomes most strongly Rayleigh-Taylor unstable because the density decreases outward steeply and thus $(d P / d r)(d \rho / d r)<0$. The instability continues to grow until the forward shock reaches the low density surface; then a rarefaction wave propagates inward from the surface to stabilize the interior. Note that in the $20 M_{\odot}$ model of SN $1987 \mathrm{~A}$ by Hachisu et al. (1990a), the most unstable is the hydrogen/helium interface due to the massive hydrogen-rich envelope. 

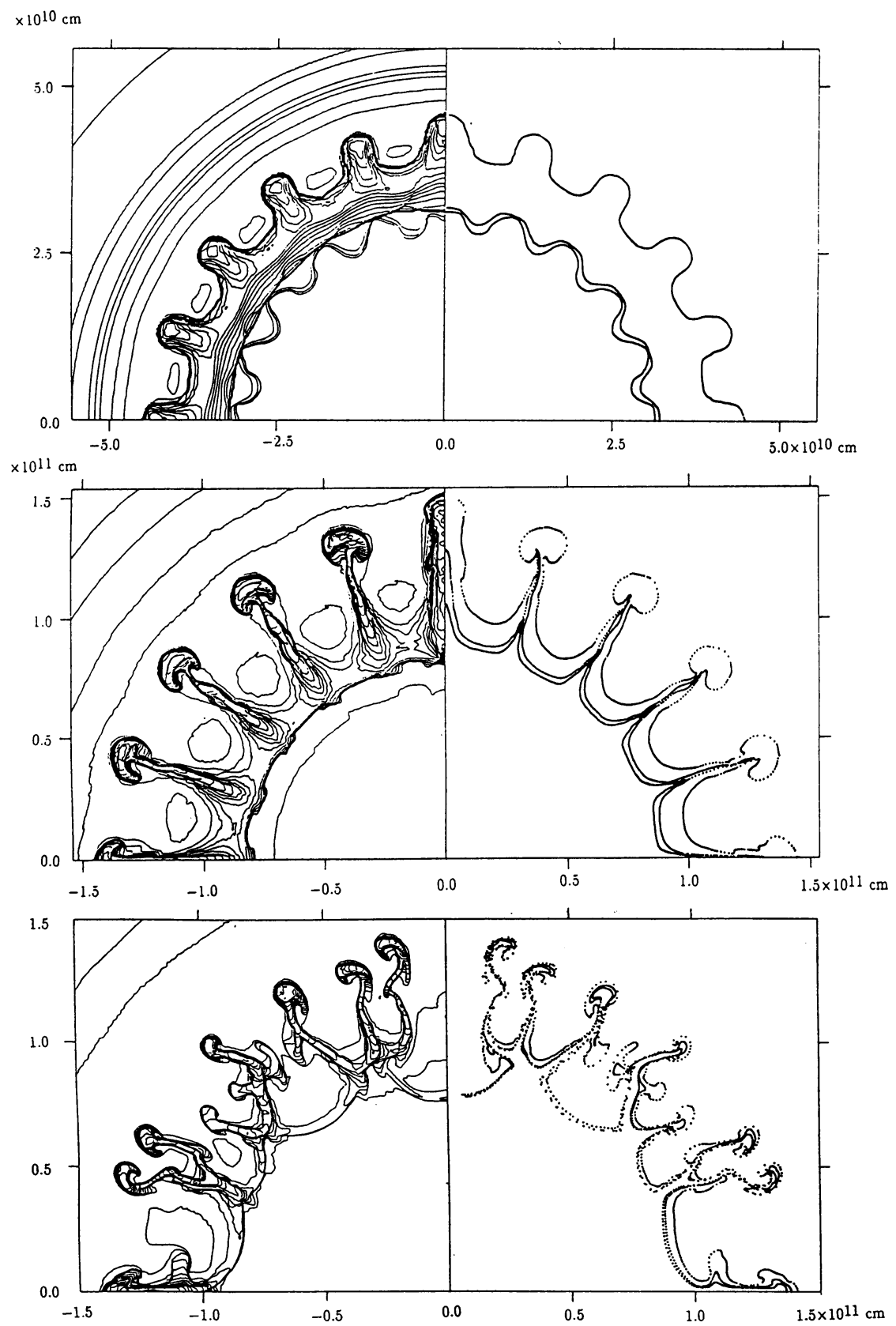

Fig. 2: Rayleigh-Taylor instabilities in the exploding helium stars of $M_{\alpha}=6 M_{\odot}$ $(t=84 \mathrm{~s})(\mathrm{a}), 4 M_{\odot}(t=200 \mathrm{~s})(\mathrm{b})$, and $3.3 M_{\odot}(t=180 \mathrm{~s})(\mathrm{c})$. Shown are the density contour map (left) and the marker particles at the composition interfaces, $\mathrm{He} / \mathrm{C}+\mathrm{O}, \mathrm{O} / \mathrm{Si}$, and $\mathrm{Ni} / \mathrm{Si}$ from the outerside (right) (Hachisu et al. 1990b). 
For the helium stars of $M_{\alpha}=3.3,4$, and $6 M_{\odot}$, Hachisu et al. (1990b) have carried out 2D hydrodynamical calculations to follow the Rayleigh-Taylor instability. As seen in Fig. 2a-c, the instability leads to only a limited mixing and clump formation for $M_{\alpha}=6 M_{\odot}$, while it does induce a large scale mixing for $M_{\alpha}=3.3$ and $4 M_{\odot}$. For $M_{\alpha}=3.3 M_{\odot},{ }^{56} \mathrm{Ni}$ is mixed to the layer of $0.4 M_{\odot}$ beneath the surface (Fig. 3), which is close to the extent of mixing as required from the light curves.

Such a mass dependence of the Rayleigh-Taylor instability can be understood from the difference in the stellar structure as follows.

1) For smaller $M_{\alpha}$ the mass ratio between the helium envelope and the core (excluding the neutron star) is larger (i.e., 2.5, 2.7, 1.0, and 0.45 for $M_{\alpha}=3.3,4,6$, and 8 $M_{\odot}$, respectively) so that the deceleration of the core and the pressure inversion are larger.

2) Smaller mass stars have steeper density gradient near the composition interface.

3) The stellar radius is larger for smaller $M_{\alpha}$, so that it takes longer for the shock wave to reach the stellar surface and the instability grows for a longer time.

We should emphasize the importance of the density structure rather than stellar mass. For example, a single Wolf-Rayet star which reduces its mass down to 4-5 $M_{\odot}$ by wind could be a SNe Ib/Ic progenitor (Langer 1989; Maeder 1990). However, such a star would not undergo extensive mixing despite the small mass, because its helium envelope would be too small to largely decelerate the core.

\section{LIGHT CURVE MODELS FOR TYPE IB SUPERNOVAE}

Figures 4 and 5 show the calculated bolometric light curves of the exploding helium stars with $M_{\alpha}=3.3,4$, and $6 M_{\odot}$ for two cases of the elemental distribution: 1) the original stratified composition structure with ${ }^{56} \mathrm{Ni}$ being confined in the innermost region (Fig. 4) and 2) the mixed distribution with almost uniform distribution of elements (Fig. 5). In order to clarify the dependence of the light curve shape on $M_{\alpha}$ and mixing, the amount of ${ }^{56} \mathrm{Ni}$ is assumed to be $0.15 M_{\odot}$ for all $M_{\alpha}$.

These figures also show three observed light curves: SN Ib 1984L (visual: Wheeler and Levreault 1985), SN Ib 1983N (bolometric: Panagia 1987), and SN Ic 1983 I (visual: Tsvetkov 1985). The visual light curve is regarded to be close to the bolometric one. The maximum luminosities and their dates are shifted to those of the corresponding calculated curves.

In the calculated bolometric light curves, an ultraviolet burst occurs at the shock breakout, but it is too brief to be observed because of the small radius of the progenitor. Later the light curve is powered by the radioactive decays of ${ }^{56} \mathrm{Ni}$ and ${ }^{56} \mathrm{Co}$. Peak luminosity is reached when the time scale of heat diffusion from the radioactive source becomes comparable to the expansion time scale. Maximum brightness is higher if the ${ }^{56} \mathrm{Ni}$ mass is larger and the date of maximum earlier. After the peak, 


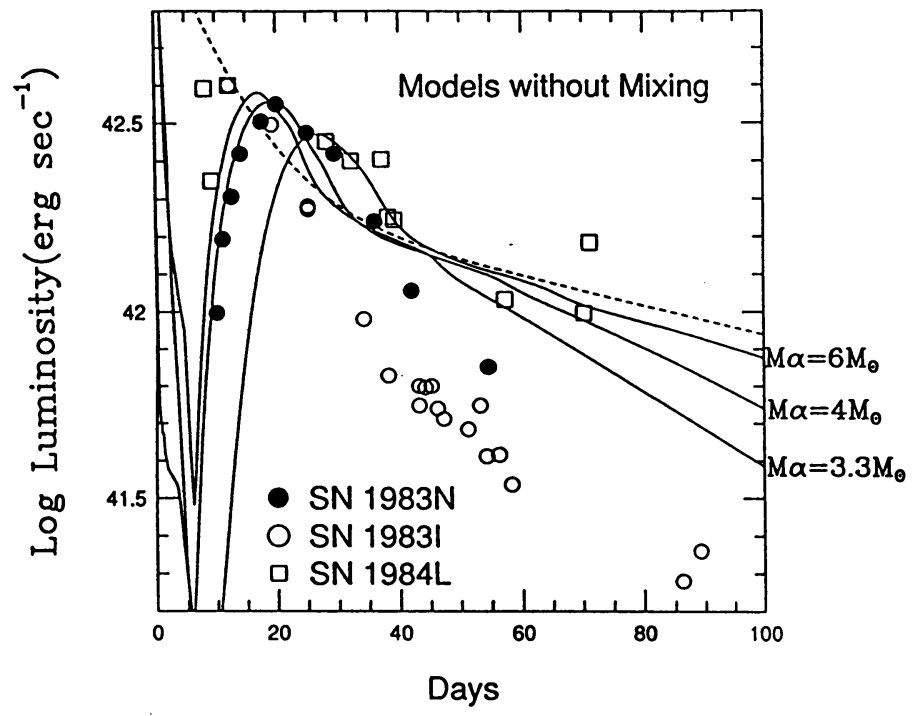

Fig. 4: Calculated light curves of exploding helium stars of $M_{\alpha}=3.3$, 4, and 6 $M_{\odot}$ (Shigeyama et al. 1990). All the models assume the production of $0.15 M_{\odot}$ ${ }^{56} \mathrm{Ni}$, kinetic energy of explosion $E=1 \times 10^{51} \mathrm{erg}$, and no mixing (i.e., stratified composition structure). Filled and open circles are the observed light curves of SN Ib 1984L (visual), 1983N (bolometric), and 1983I (visual). The dotted curve is the energy generation rate of the ${ }^{56} \mathrm{Ni}^{-56} \mathrm{Co}$ decays.

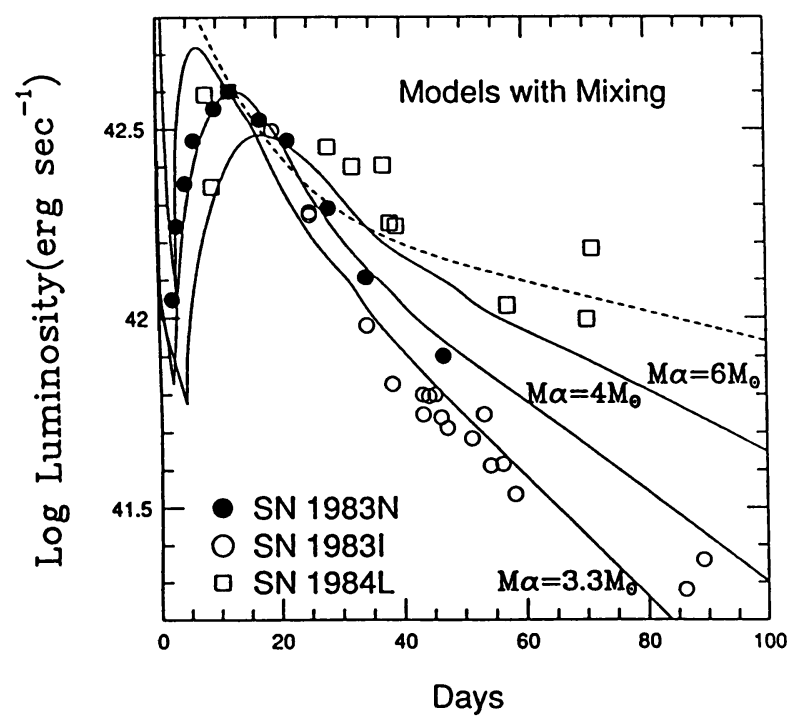

Fig. 5: Same as Fig. 4 but with mixed abundance distribution similar to Fig. 3. 
the optical light curve declines at a rate that depends on how fast gamma-rays from the radioactive decays escape from the star without being thermalized. Since the escape probability of gamma rays is determined by the column depth to the $\mathrm{Ni}-\mathrm{Co}$ layer, the optical light curve declines faster if the ejected mass is smaller and if ${ }^{56} \mathrm{Ni}$ is mixed closer to the surface.

For the unmixed cases in Fig. 4, the calculated light curve tail declines faster for smaller $M_{\alpha}$. However, these tails (even the fastest decline of $M_{\alpha}=3.3 M_{\odot}$ ) are much slower than that of SN $1983 \mathrm{~N}$ and $83 \mathrm{I}$ after $\sim$ day 40 .

For the mixed cases in Fig. 5, the light curve shape is significantly different from the unmixed cases:

1) The maximum luminosity is reached $\sim 10$ days earlier and thus being higher than in the unmixed cases because of the earlier radioactive heating of the surface layers.

2) The decline of the tail is much faster than the unmixed cases.

Figure 5 shows that the bolometric light curve of $M_{\alpha}=4 M_{\odot}$ is in excellent agreement with SN Ib $1983 \mathrm{~N}$ from the pre-maximum through day 50 . The calculated curve for 50-100 days continues to be close to the bolometric light curve obtained by Leibundgut (1988). Other well-observed $\mathrm{SNe} \mathrm{Ib}$ generally have early-time visual and bolometric light curves whose shapes are nearly identical to those of SNe Ia (Porter and Filippenko 1987; Leibundgut 1988). On the other hand, the light curve of the mixed 3.3 $M_{\odot}$ model, rising and declining faster than $\mathrm{SN} 1983 \mathrm{~N}$, is in good agreement with SN Ic 1983I (see the next section).

\section{LIGHT CURVE MODELS FOR TYPE IC SUPERNOVAE}

As mentioned in $\S 1$, the light curve of SN Ic $1987 \mathrm{M}$ provided new constraints on the models (Nomoto et al. 1990). In Fig. 6 the filled circles show a quasi-bolometric (3280-9100 $\AA$ ) light curve constructed from flux-calibrated spectra, where maximum brightness is assumed to be subluminous relative to SNe Ia by $0.6 \mathrm{mag}$ as observed in the $B$ band (Filippenko et al. 1990). Figure 6 also shows the observed bolometric curves of SNe Ia 1972E and 1981B, and of SN Ib 1983N for comparison. In each case, the observed light curve has been shifted along the abscissa to match the corresponding theoretical curve. The peak bolometric luminosities of the two SNe Ia are assumed to match the theoretical predictions of model W7 which corresponds to $H_{0}=60 \mathrm{~km} \mathrm{~s}^{-1} \mathrm{Mpc}^{-1}$ (Arnett et al. 1985; Nomoto 1986).

Figure 6 demonstrates two important features of SN Ic 1987M: 1) its brightness fell somewhat more rapidly than that of SNe Ia and SN Ib 1983N, and 2) SN 1987M was considerably more luminous than SN 1983N, which was $\sim 1.5$ mag fainter than SNe Ia at maximum (Panagia 1987). These features are very difficult to understand with the previous massive helium star models.

In Fig. 6, illustrated is the light curve of the exploding $3.3 M_{\odot}$ helium star with 


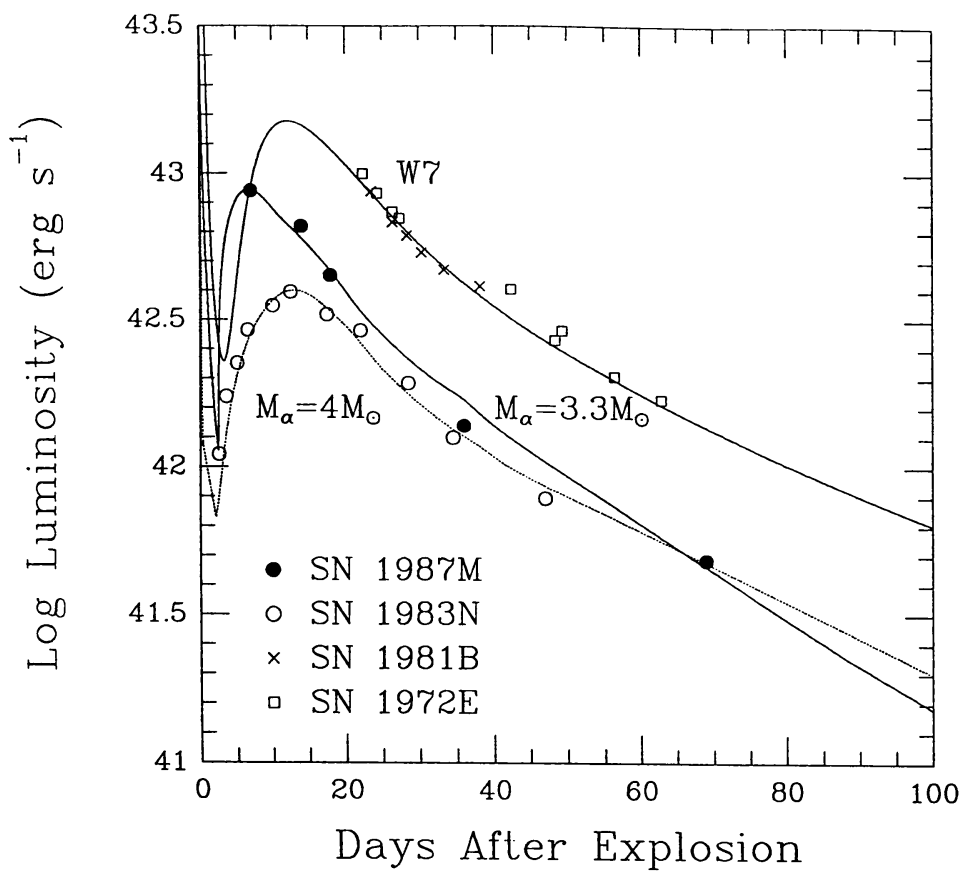

Fig. 6: Quasi-bolometric ( $\lambda \lambda 3280-9100)$ light curve of SN Ic 1987M, together with the bolometric light curves of SNe Ia 1981B and 1972E (Graham 1987) and of SN Ib $1983 \mathrm{~N}$ (Panagia 1987). The predicted curves of the $3.3 M_{\odot}$ model for SN $1987 \mathrm{M}$, the $4 M_{\odot}$ model for SN Ib, and the W7 model for SN Ia are indicated by solid and dotted lines (Nomoto et al. 1990).

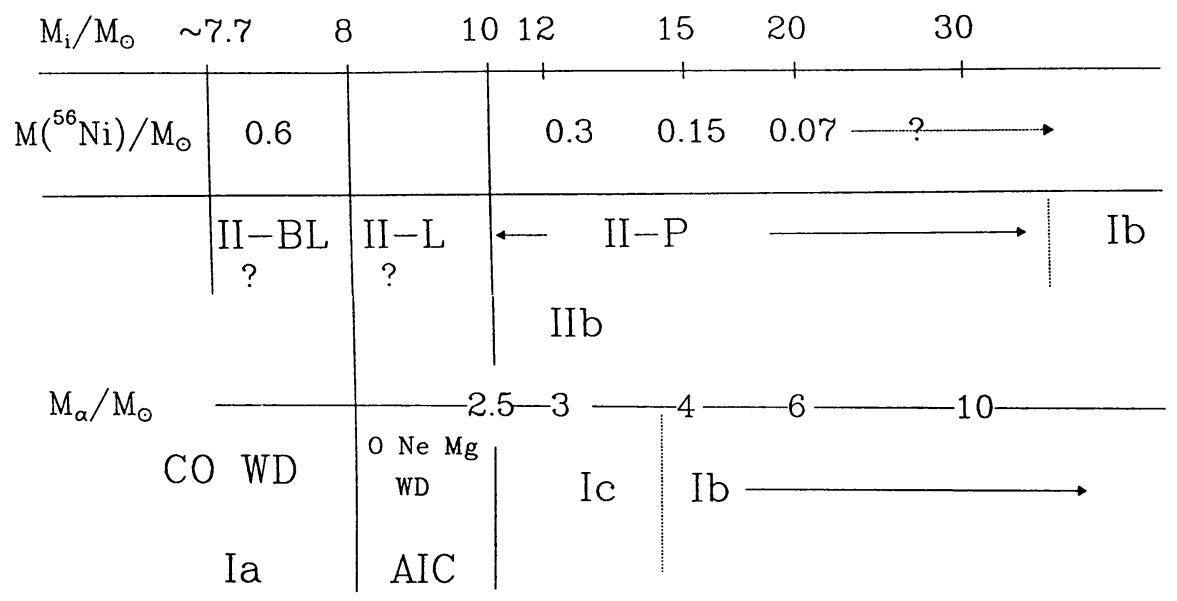

Fig. 7: Hypothetical connection between supernova types and their progenitors for single stars (upper) and close binary stars (lower). $M_{\mathrm{i}}$ and $M_{\alpha}$ are the initial mass and the helium star mass, respectively. AIC stands for accretion-induced collapse of white dwarfs. 
$0.26 M_{\odot}$ of ${ }^{56} \mathrm{Ni}$ being mixed almost uniformly from the center through the layer at $0.2 M_{\odot}$ beneath the surface. It is clear that the calculated bolometric light curve of the 3.3 $M_{\odot}$ model closely matches the quasi-bolometric curve of SN $1987 \mathrm{M}$, both in shape and in peak luminosity. Figure 6 also shows the $4 M_{\odot}$ helium star model for $\mathrm{SNe} \mathrm{Ib}$ (where the ${ }^{56} \mathrm{Ni}$ mass is $0.15 M_{\odot}$ ) and the white dwarf model W7 for SNe Ia (where the ${ }^{56} \mathrm{Ni}$ mass is $0.58 M_{\odot}$; Nomoto et al. 1984).

Compared with the $4 M_{\odot}$ model for $\mathrm{SNe} \mathrm{Ib}$, the 3.3 $M_{\odot}$ model has several attractive features for SN 1987M. First, maximum brightness is higher by more than a factor of two because of the larger ${ }^{56} \mathrm{Ni}$ mass and the earlier date of maximum brightness. Second, decline in the tail is noticeably faster due to the more extensive mixing and smaller ejected mass. Compared with the W7 model for SNe Ia, the 3.3 $M_{\odot}$ model gives a lower maximum brightness (by about $0.6 \mathrm{mag}$ ) and a faster decline, just as observed in SN 1987M.

\section{PROGENITORS OF TYPE IB/IC/IIB SUPERNOVAE}

Figure 7 summarizes the currently proposed initial masses $M_{\mathrm{i}}$ of the progenitors for the various types of supernovae. The upper and lower rows respectively show the cases of single stars and helium stars of masses $M_{\alpha}$ (or white dwarfs) in close binary stars. The produced masses of ${ }^{56} \mathrm{Ni}$ inferred from light curves are also given.

The main conclusion of the previous sections shown in Fig. 7 is that typical $\mathrm{SNe} \mathrm{Ib}$ and Ic are the explosions of helium stars of 3-5 $M_{\odot}$ in close binary systems (see also Uomoto 1986). The peak luminosities and the fast decline of the light curves of $\mathrm{SNe} \mathrm{Ib} / \mathrm{Ic}$ can be well modeled because these low mass stars 1) eject smaller mass, 2) undergo more extensive mixing, and 3) synthesize larger amount of ${ }^{56} \mathrm{Ni}$. In particular, the light curve of SN Ic 1987M supports the theoretical prediction (Shigeyama et al. 1990) that low-mass helium stars in close binary systems produce larger amounts of ${ }^{56} \mathrm{Ni}$ than do high-mass helium stars.

Helium stars with 3-5 $M_{\odot}$ can form from stars of 12-18 $M_{\odot}$ after Roche-lobe overflow in close binary systems. The fact that SN Ic $1987 \mathrm{M}$ was superposed on a spiral arm of NGC 2715, but not on a very luminous H II region (Filippenko et al. 1990), is consistent with a progenitor having this initial mass. It is unlikely that these supernova progenitors would have been classified as Wolf-Rayet stars with broad emission lines, since their mass-losing wind was probably weak.

Radio observations of some.SNe Ib/Ic 1983N, 1984L, and 1990B imply that the progenitors of these supernovae had dense circumstellar shells (Sramek et al. 1984). In the 3-5 $M_{\odot}$ helium star models, mass exchange during the early binary evolution may make the companion a massive OB star. The wind from such a companion star could interact with the progenitor's wind to form a dense circumstellar shell around the helium star progenitor. Also some gas could have been left after previous episodes of mass exchange. 
For larger $M_{\alpha}$, helium stars of $M_{\alpha} \sim 6 M_{\odot}$ produces too small amount of ${ }^{56} \mathrm{Ni}$ $\left(\sim 0.07 M_{\odot}\right)$ to be consistent with SNe Ib. More massive helium stars might produce larger amount of ${ }^{56} \mathrm{Ni}$. Also extensive mass loss from very massive stars could result in the formation of Wolf-Rayet stars with final masses as small as 4-5 $M_{\odot}$ (Langer 1989; Maeder 1990). However, such a progenitor does not undergo a strong RayleighTaylor instability, since its helium envelope is not sufficiently massive to greatly decelerate the core. Without mixing and the associated formation of clumps, the resulting light curve is too broad to explain SNe Ib/Ic. Furthermore, the birth rate of very massive stars is too small to explain the occurrence frequency of $\mathrm{SNe} \mathrm{Ib} / \mathrm{Ic}$ (Evans et al. 1989).

Figure 7 suggests that $\mathrm{SNe} \mathrm{Ib} / \mathrm{Ic}$ and typical Type II-P supernovae (SNe II-P) originate from the similar stellar mass range, where $\mathrm{SNe} \mathrm{Ib}$ are from close binaries while SNe II-P are single stars. It is suggestive that the light curve tail of the best observed SN II-P 1969L follows the ${ }^{56}$ Co decay rate and the inferred ${ }^{56} \mathrm{Ni}$ mass is $0.13 M_{\odot}$ if $H_{0} \sim 60$ (Kirshner and Kwan 1974); this is close to the ${ }^{56} \mathrm{Ni}$ mass from $\mathrm{SNe} \mathrm{Ib}$.

It is interesting to compare $\mathrm{SNe} \mathrm{Ib/Ic} \mathrm{and} \mathrm{SN} 1987 \mathrm{~K}$ whose spectral classification changed from Type II to Type Ib/Ic as it aged, thereby being called as SNe IIb (Filippenko 1988). The decline of the SN 1987K light curve is as fast as SNe Ia (and thus SNe Ic) (Turatto et al. 1990). The early time spectra of SN $1987 \mathrm{~K}$ are very similar to SNe Ic (1983V and $87 \mathrm{M})$, and, very recently, hydrogen feature is identified in the early time spectrum of SN Ic 1987M (Jeffery and Branch 1990). This strongly suggests that the difference in the spectral feature between $\mathrm{SNe} I c$ and $\mathrm{Ib}$ is due to the presence of a thin envelope of hydrogen in SNe Ic immediately prior to the explosion. Some hydrogen can be left on the helium star after mass exchange for a certain combination of the initial mass and the close binary separation (Yamaoka and Nomoto 1990).

To examine the above hypothesis, theoretical spectra should take into account non-LTE excitation of hydrogen and helium lines due to mixed radioactive materials (Branch 1988; Wheeler and Harkness 1990). Differences in relative distribution of $\mathrm{H}, \mathrm{He}$, and ${ }^{56} \mathrm{Ni}$ and their amounts after mixing might be related to the spectral differences among $\mathrm{SNe} \mathrm{Ib}$, Ic, and IIb.

White dwarfs have also occasionally been considered as progenitors of SNe Ic. However, the light curve produced by the off-center single detonation model (Branch and Nomoto 1986; Nomoto 1982; Woosley et al. 1986) declines too fast. The carbon deflagration model with slow flame speed (Woosley 1990) releases smaller kinetic energy than do SNe Ia, thereby forming a light curve which declines more slowly than that of SNe Ia. This appears to be inconsistent with observations of SN 1987M (Fig. 4). Therefore supernova explosions of accreting $\mathrm{C}+\mathrm{O}$ white dwarfs become mostly $\mathrm{SNe} \mathrm{Ia}$ and $\mathrm{O}+\mathrm{Ne}+\mathrm{Mg}$ white dwarfs undergo accretion-induced collapse (AIC). 
SNe II-L (linear) and II-BL (bright linear, like SN 1979C; Branch, private communication) do not show a plateau, thereby being suggested to have hydrogen-rich envelope of smaller than $\sim 1 M_{\odot}$. In Fig. 7, SNe II-BL and II-L are tentatively assumed to be the explosions of AGB stars having degenerate $\mathrm{C}+\mathrm{O}$ cores (carbon deflagration) and $\mathrm{O}+\mathrm{Ne}+\mathrm{Mg}$ cores (electron capture collapse), respectively (Swartz et al. 1990), since the AGB stars have lost most of their hydrogen-rich envelopes at the explosion. However, the progenitors having small hydrogen might also be related to the Wolf-Rayet stars.

I would like to thank T. Shigeyama, I. Hachisu, T. Matsuda, T. Tsujimoto, M. Hashimoto, F.-K. Thielemann, and A.V. Filippenko, for collaborative work on $\mathrm{SNe} \mathrm{Ib} / \mathrm{Ic}$. I am also grateful to D. Branch and J.C. Wheeler for stimulating discussion on this subject. This work has been supported in part by the grant-in-Aid for Scientific Research (01540216, 01790169, 02234202, 02302024) of the Ministry of Education, Science, and Culture in Japan, and by the Japan-U.S. Cooperative Science Program (EPAR-071/88-15999) operated by the JSPS and the NSF.

\section{REFERENCES}

Arnett, W.D., Branch, D., Wheeler, J.C. 1985, Nature 314, 337

Arnett, W. D., Fryxell, B. A., and Müller, E. 1989, Ap. J. (Letters), 341, L63.

Begelman, M.C., and Sarazin, C.L. 1986, Ap. J. (Letters), 302, L59.

Branch, D. 1986, Ap. J. (Letters), 300, L51.

. 1988, in IAU Colloquium 108, Atmospheric Diagnostics of Stellar Evolution, ed. K. Nomoto, Lecture Notes in Physics, 305, 281.

Branch, D., and Nomoto, K. 1986, Astr. Ap., 164, L13.

Den, M., Yoshida, T., and Yamada, Y. 1990, preprint (KUNS1004).

Evans, R., van den Bergh, S., McClure, R.D. 1989, Ap. J. 345, 752

Ensman, L., and Woosley, S.E. 1988, Ap. J., 333, 754.

Filippenko, A.V. 1988, Astr. J., 96, 1941.

Filippenko, A.V., Porter, A.C., and Sargent, W.L.W. 1990, Astr. J., in press.

Filippenko, A.V., and Sargent, W.L.W. 1989, Ap. J. (Letters), 345, L43.

Fransson, C., and Chevalier, R.A. 1989, Ap. J., 343, 323.

Fryxell, B.A., Arnett, W.D., and Müller, E. 1991, Ap. J., in press.

Gaskell, C.M., Cappellaro, E., Dinerstein, H.L., Garnett, D.R., Harkness, R.P., and Wheeler, J.C. 1986, Ap. J. (Letters), 306, L77.

Graham, J.R. 1987, Ap. J., 315, 588.

Hachisu, I., Matsuda, T., Nomoto, K., and Shigeyama, T. 1990a, Ap. J. (Letters), 358, L57 1990b, Ap. J. (Letters), in press.

Harkness, R.P., and Wheeler, J.C. 1990, in Supernovae, ed. A. Petschek (SpringerVerlag), p. 1. 
Harkness, R.P. et al. 1987, Ap. J., 317, 355.

Hashimoto, M., Nomoto, K., and Shigeyama, T. 1988, Astr. Ap., 210, L5.

Jeffery, D., and Branch, D. 1990, private communication.

Kirshner, R.P., and Kwan, J. 1974, Ap. J., 193, 27.

Kumagai, S., Shigeyama, T., Nomoto, K., Itoh, M., Nishimura, J., and Tsuruta, S. 1989, Ap. J., 345, 412.

Langer, N. 1989, Astr. Ap., 220, 135.

Leibundgut, B. 1988, Ph.D. thesis, Universität Basel.

Maeder, A. 1990, Astr. Ap. Suppl., in press.

Nomoto, K. 1982, Ap. J., 257, 780.

- 1986, Ann. New York Acad. Sci. 470, 294

Nomoto, K., Filippenko, A.V., and Shigeyama, T. 1990, Astr. Ap., submitted.

Nomoto, K., and Hashimoto, M. 1988, Physics Reports, 163, 13.

Nomoto, K., Shigeyama, T., and Hashimoto, M. 1988a, in IAU Colloquium 108, Atmospheric Diagnostics of Stellar Evolution, ed. K. Nomoto, Lecture Notes in Physics, 305, 319.

Nomoto, K., Shigeyama, T., Kumagai, S., and Hashimoto, M. 1988b, in Proc. Astr. Soc. Australia, 7, 490.

Panagia, N. 1987, in High Energy Phenomena Around Collapsed Stars, ed. F. Pacini (D. Reidel), p. 33.

Porter, A.C., and Filippenko, A.V. 1987, Astr. J. 93, 1372

Schaeffer, R., Casse, M., and Cahen, S. 1987, Ap. J., 316, L31.

Schlegel, E.M., and Kirshner, R.P. 1989, Astr. J., 98, 577.

Shigeyama, T., Nomoto, K., and Hashimoto, M. 1988, Astr. Ap., 196, 141.

Shigeyama, T., Nomoto, K., Tsujimoto, T., and Hashimoto, M. 1990, Ap. J. (Letters), in press.

Sramek, R.A., Panagia, N., and Weiler, K.W. 1984, Ap. J. (Letters), 285, L59.

Swartz, D.A., Wheeler, J.C., and Harkness, R.P., 1990, Ap. J., submitted.

Thielemann, F.-K., Hashimoto, M., and Nomoto, K. 1990, Ap. J., 349, 222.

Tsvetkov, D.Yu. 1985, Sov. Astr., 29, 211.

Turatto, M., Cappellaro, E., Barbon, R., Della Valle, M., Ortolani, S., and Rosino, L. 1990, Astr. J., in press.

Uomoto, A. 1986, Ap. J. (Letters), 310, L35.

Uomoto, A., and Kirshner, R.P. 1985, Astr. Ap., 149, L7.

Wheeler, J.C., and Levreault, R. 1985, Ap. J. (Letters), 294, L17.

Wheeler, J.C., and Harkness, R. 1990, Phys. Rep., in press.

Woosley, S.E. 1988, Ap. J., 330, 218.

- 1990, In Supernovae, ed. A. Petschek (Springer-Verlag), p. 182.

Woosley, S.E., Taam, R.E., and Weaver, T.A. 1986, Ap. J., 301, 601.

Yamada, Y., Nakamura, T., and Oohara, K. 1990, preprint (KUNS1019).

Yamaoka, H., and Nomoto, K. 1990, in preparation. 


\section{DISCUSSION}

Vanbeveren: A star $\left(\sim 13 M_{\odot}\right)$ in a close binary still has some hydrogen in the outer layers after the Roche lobe overflow process. Can you make a rough guess how much $H$ may still be present in order not to see it during the SN?

Nomoto: From the similarity between the early time spectrum of Type Ic SN1987M and that of Type IIb SN1987K, I have speculated that a small amount of hydrogen might exist in Type Ic supernovae. That is why I speculate that SN IIb might also originate from low-mass helium stars. Either hydrogen or mixed heavy elements may be the source of difference between the Ic and Ib spectra. Non-LTE calculation of synthetic spectra with non-thermal radioactive heating is necessary to estimate a limit to the hydrogen mass.

Shara: How large were the asymmetries and/or perturbations you assumed in order to get the plumes you showed?

Nomoto: We applied two types of perturbations, a periodic sinusiodal one and a random one, to the velocity by 5 percent. We are now studying how the results depend on the magnitude and wavelength of perturbation with more accurate methods.

Matteucci: What is the physical reason for having the amount of iron produced by massive stars decreasing with initial stellar mass?

Nomoto: For the evolution of smaller mass stars the effect of Coulomb interaction to reduce pressure is larger, which eventually makes the iron core mass smaller (as small as $1.18 M_{\odot}$ for the $13 M_{\odot}$ star. This results in the formation of a more massive $S i$-rich layer above the iron core. If the neutron star mass is close to the iron core mass at the explosion, larger mass of ${ }^{56} \mathrm{Ni}$ is produced from the $S i$-rich layer. This is the case for the $12-16 M_{\odot}$ stars. However, the mass of ejected iron depends strongly on the mass cut that divides the neutron star and the ejecta, which can be constrained only by the observations until the explosion mechanism becomes clear. The rather small mass of ${ }^{56} \mathrm{Ni}$ in SN1987A and the faintness of the Cas A explosion suggest that the mass cut could be significantly larger than the iron core mass for stars more massive than $20 M_{\odot}$.

Maeder: I would appreciate a few comments from you regarding the observed abundances in SN1987A and how they compare with your nucleosynthetic yields.

Nomoto: The abundances obtained from explosive nucleosynthesis calculation for the $20 M_{\odot}$ model of SN1987A (Nomoto et al., 1990) are in good agreement with the abundances determined from the ESO observations (Dantziger, 1990). 